Supplement of Clim. Past, 15, 1825-1844, 2019

https://doi.org/10.5194/cp-15-1825-2019-supplement

(C) Author(s) 2019. This work is distributed under

the Creative Commons Attribution 4.0 License.

(c) (i)

Supplement of

\title{
Identifying teleconnections and multidecadal variability of East Asian surface temperature during the last millennium in CMIP5 simulations
}

Satyaban B. Ratna et al.

Correspondence to: Satyaban B. Ratna (s.bishoyi-ratna@uea.ac.uk)

The copyright of individual parts of the supplement might differ from the CC BY 4.0 License. 


\section{Sensitivity test: omitting the Historical (1850-2000) simulations}

The analysis in the main text uses data the Last Millennium (LM) and Historical (H) forced simulations concatenated to form a continuous series from 850 to 2000. Prior to concatenation, each series (LM and H) is linearly detrended to remove any minor residual drift in the LM series and to remove trends driven by increasing anthropogenic forcing during the historical period (because our focus is on variability rather than global warming trends). Linearly detrending the $\mathrm{H}$ simulations may not be ideal, however, because the anthropogenic forcing is not linear and the diagnosis of modes of variability can be affected as a result (Steinman et al., 2015). We assess the potential effect on our results by repeating them using only the LM simulations (Figures S1 and S2). Our results are not sensitive to this choice. The correlations between the AMO and East Asian temperature (Figure S1) are very similar if we just use LM compared to those from the combined LMH simulations, showing the same sensitivity to AMO definition (much weaker correlations for AMOr than for AMOnr) and the same behaviour between GCMs (e.g. weakest correlations for BCC, strongest for CCSM4). There are some secondary differences (most notably for MRI which shows different strength correlations with AMOr in the NW of our East Asian study area) but these do not alter our overall conclusion. An advantage of using the LMH series is that it is longer and therefore slightly more powerful at identifying whether relatively weak correlations are statistically difference from zero. This is apparent from the slightly different position of the significance contours in Figures S1 and S2, and this is the main difference between the LM and LMH correlations between the PDO and East Asian temperatures (Figure S2). 

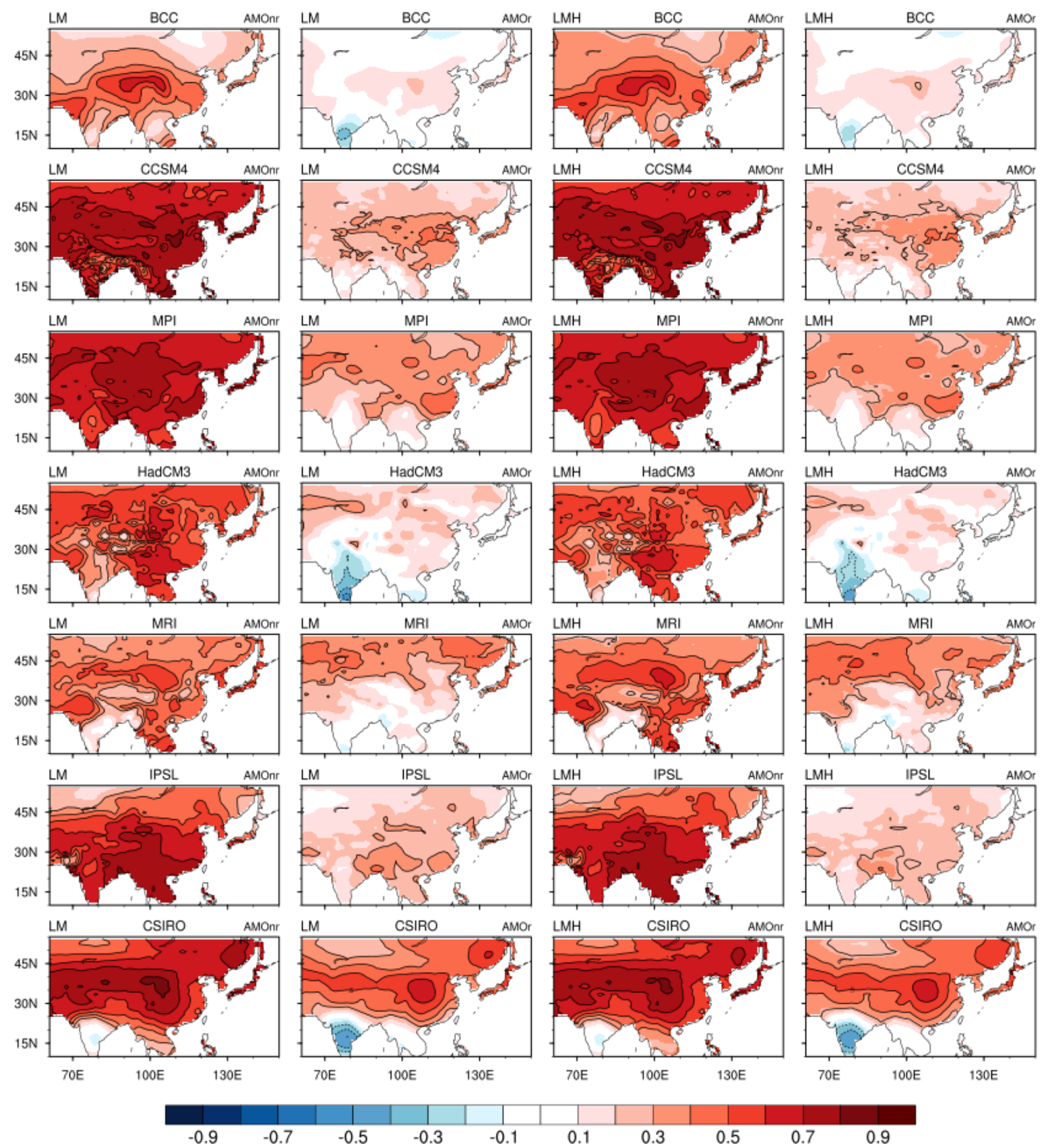

Figure S1: As Fig. 3 but for the comparison between Last Millennium (850-1849) simulation (columns 1 and 2) and combined Last Millennium plus historical (850-2000) simulations (columns 3 and 4). 

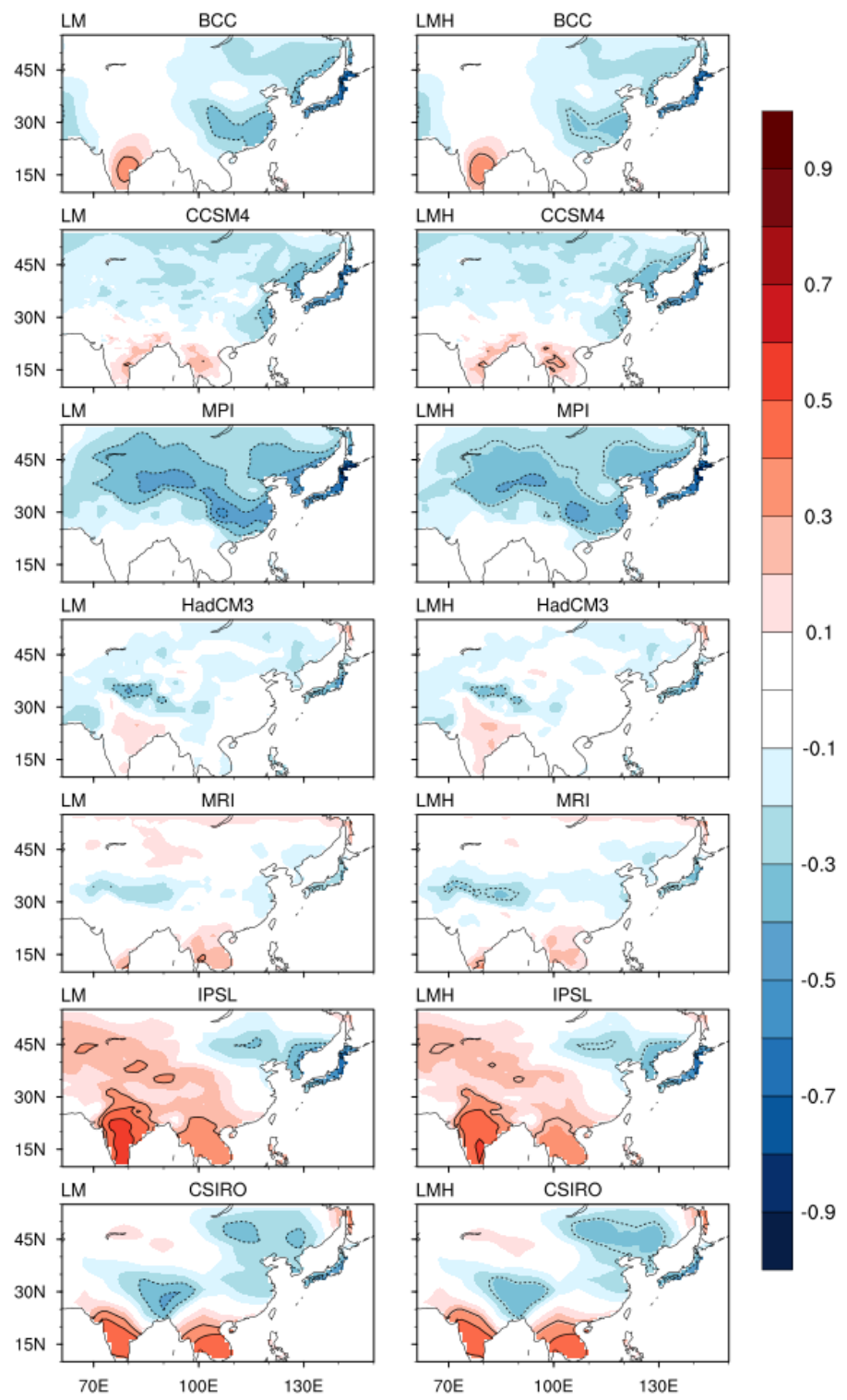

Figure S2: As Fig.5 but for the comparison between Last Millennium (850-1849) simulation (column 1) and combined Last Millennium plus historical (850-2000) simulations (column 2). 


\section{Sensitivity test: individual East Asian temperature reconstructions}

The East Asian temperature reconstruction we used, from Wang et al. (2018), is a composite of seven published reconstructions. Due to differences in the underlying proxy data used (e.g. the use of annually-resolved tree-ring data) the ability of each reconstruction to capture the response to forcings may differ. In case some of our findings are sensitive to the choice of reconstruction we have recalculated the correlations using three of the individual summer temperature reconstructions (CK13, Cook et al. 2013; Shi15, Shi et al. 2015; ZN18, Zhang et al. 2018) within the Wang et al. (2017) composite. A comparison of these time series can be seen in Wang et al (2018).

Correlations between these East Asian temperature reconstructions and AMO (Wang et al. 2017, Mann et al. 2009), PDO (Mann et al. 2009, MacDonald et al. 2005), volcanic (GRA, Gao et al. 2008; CEA, Crowley et al. 2008; SIG, Sigl et al. 2015) and solar forcing (VSK, Vieira et al. 2011; DB, Delaygue and Bard (2011); SBF; Steinhilber et al. 2009) are given in Figure S3. There are some interesting differences between the Wang et al. (2018) composite and the three individual reconstructions, perhaps related to how well they resolve the response to volcanic forcing. However, with the exception of the correlations between East Asian temperature and the Mann et al. (2009) AMO index, the only significant correlations (with some solar forcing, PDO and AMO reconstructions) at the multidecadal timescales are with the Wang et al. (2018) composite reconstruction. Cook et al. (2013) report a volcanic cooling signal in their temperature reconstruction. Correlations with volcanic forcing are slightly stronger for Cook et al. (2013) than for the other reconstructions but they are still not statistically significant at the multidecadal timescale. This contrasts with six of the seven climate models, which do show significant multidecadal correlations between simulated East Asian $\mathrm{T}$ and volcanic forcing. The volcanic influence on the reconstructed temperatures is limited to the interannual timescale.

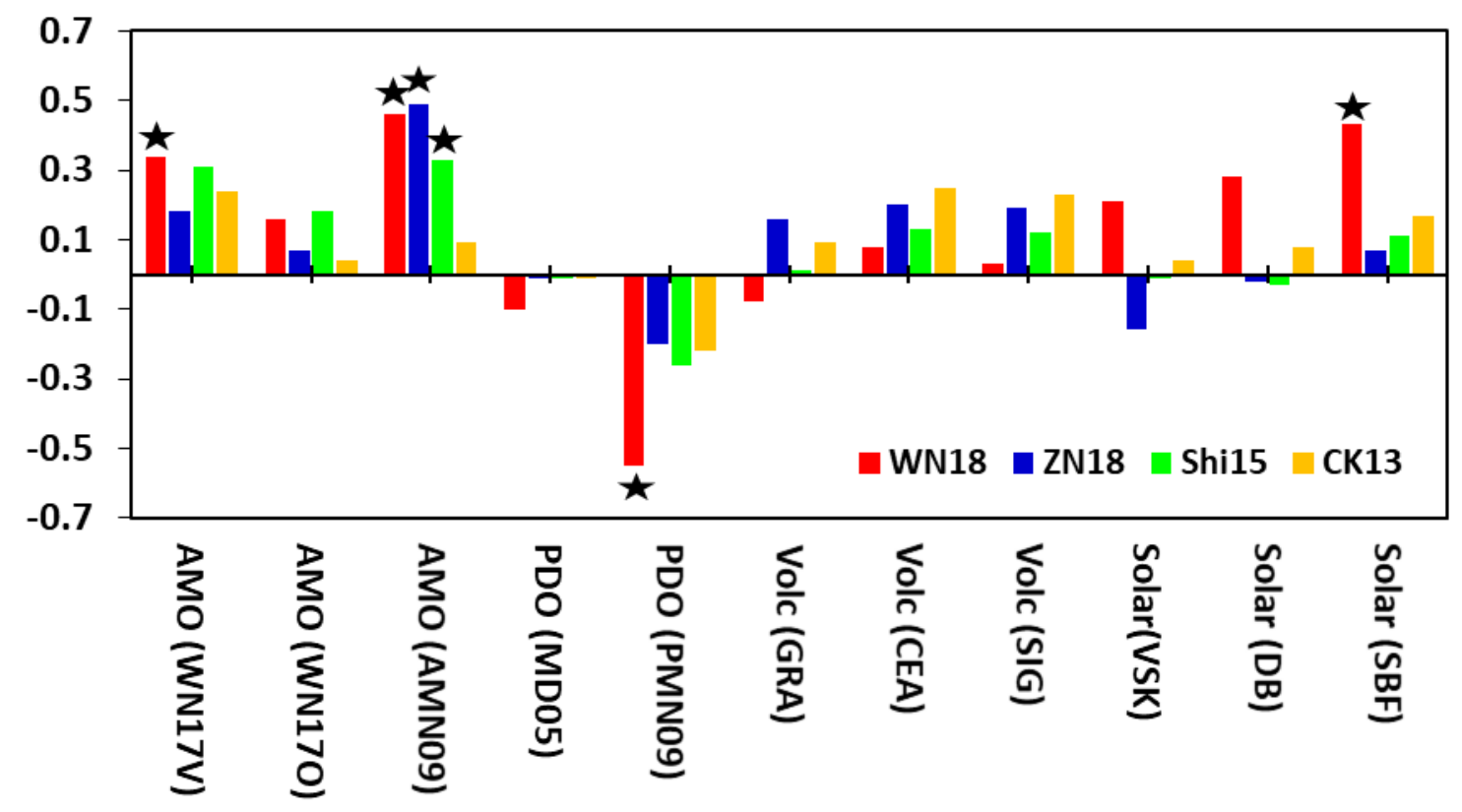

Figure S3: Correlation of different East Asian TAS reconstructions (the composite of Wang et al., 2018, plus three components of this composite: Cook et al. 2013, Shi et al. 2015, Zhang et al. 2018) with AMO, PDO, volcanic forcing and solar forcing reconstructions. The bars marked with 'star' are significant at 95\% level using a twotailed student $\mathrm{t}$-test. 